\title{
Controllability of Fractional Neutral Stochastic Integro-Differential Systems with Infinite Delay
}

\author{
Xichao Sun, ${ }^{1}$ Litan Yan, ${ }^{2}$ and Jing $\mathrm{Cui}^{3}$ \\ ${ }^{1}$ College of Information Science and Technology, Donghua University, 2999 North Renmin Road, Songjiang, Shanghai 201620, China \\ ${ }^{2}$ Department of Mathematics, Donghua University, 2999 North Renmin Road, Songjiang, Shanghai 201620, China \\ ${ }^{3}$ Department of Mathematics, Anhui Normal University, 1 East Beijing Road, Wuhu 241000, China
}

Correspondence should be addressed to Litan Yan; litanyan@dhu.edu.cn

Received 28 October 2012; Revised 20 January 2013; Accepted 27 January 2013

Academic Editor: Engang Tian

Copyright (C) 2013 Xichao Sun et al. This is an open access article distributed under the Creative Commons Attribution License, which permits unrestricted use, distribution, and reproduction in any medium, provided the original work is properly cited.

This paper is concerned with the controllability of a class of fractional neutral stochastic integro-differential systems with infinite delay in an abstract space. By employing fractional calculus and Sadovskii's fixed point principle without assuming severe compactness condition on the semigroup, a set of sufficient conditions are derived for achieving the controllability result.

\section{Introduction}

It is well known that the fractional calculus is a classical mathematical notion and is a generalization of ordinary differentiation and integration to arbitrary (noninteger) order. Nowadays, studying fractional-order calculus has become an active research field [1-7]. Much effort has been devoted to apply the fractional calculus to networks control. For example, Chen et al. [8], Delshad et al. [9], and Wang and Zhang [10] studied the synchronization for fractional-order complex dynamical networks; Zhang et al. [11] investigated a fractional order three-dimensional Hopfield neural network and pointed out that chaotic behaviors can emerge in a fractional network; Kaslik and Sivasundaram [12] discussed the local stability for fractional-order neural networks of Hopfield type by applying the linear stability theory of fractional-order system.

One of the emerging branches of this study is the theory of fractional evolution equations, say, evolution equations, where the integer derivative with respect to time is replaced by a derivative of fractional order. The increasing interest in this class of equations is motivated both by their application to problems from fluid dynamic traffic model, viscoelasticity, heat conduction in materials with memory, electrodynamics with memory, and also because they can be employed to approach nonlinear conservation laws (see [13] and references therein). In addition, neutral stochastic differential equations with infinite delay have become important in recent years as mathematical models of phenomena in both science and engineering, for instance, in the theory development in Gurtin and Pipkin [14] and Nunziato [15] for the description of heat conduction in materials with fading memory. It should be pointed out that the deterministic models often fluctuate due to noise, which is random or at least appears to be so. Therefore, we must move from deterministic problems to stochastic ones. We mention here the recent papers $[16,17]$ concerning the existence of mild solutions of fractional stochastic systems.

As one of the fundamental concepts in mathematical control theory, controllability plays an important role both in deterministic and stochastic control problems such as stabilization of unstable systems by feedback control. Roughly speaking, controllability generally means that it is possible to steer a dynamical control system from an arbitrary initial state to an arbitrary final state using the set of admissible controls. Controllability problems for different nonlinear stochastic systems in infinite dimensional spaces have been extensively studied in many papers; see [18-22] and references therein. We would also like to mention that the controllability for stochastic systems with infinite delay has been investigated by Balasubramaniam et al. $[23,24]$ and Ren et al. [25] using some abstract spaces. Nevertheless, to 
the best of our knowledge, it seems that little is known about the controllability of fractional neutral stochastic differential equations with infinite delay, and the aim of this paper is to close this gap.

In this paper, we are interested in the controllability of a class of fractional neutral stochastic integro-differential systems with infinite delay of the followin form:

$$
\begin{gathered}
\frac{{ }^{c} D^{\alpha}}{d t^{\alpha}}\left[x(t)+G\left(t, x_{t}\right)\right]= \\
\quad+A x(t)+B u(t) \\
\quad+\int_{0}^{t} \sigma\left(t, s, x_{s}\right) d W(s), \quad t \in J, \\
x(t)=\phi(t), \quad t \in(-\infty, 0] .
\end{gathered}
$$

Here, $J:=[0, b], b>0 . x(\cdot)$ takes value in a real separable Hilbert space $\mathbb{H}$ with inner product $(\cdot, \cdot)$ and norm $|\cdot|_{\mathbb{H}}$. The fractional derivative ${ }^{c} D^{\alpha}, \alpha \in(1 / 2,1]$ is understood in the Caputo sense. $-A: \mathscr{D}(-A) \subset \mathbb{M} \rightarrow \mathbb{W}$ is the infinitesimal generator of a analytic semigroup of a bounded linear operator $S(t), t \geq 0$, on $\mathbb{U}$. Let $\mathbb{K}$ be another separable Hilbert space with inner product $(\cdot, \cdot)_{\mathbb{K}}$ and norm $\|\cdot\|_{\mathbb{K}} \cdot W$ is a given $\mathbb{K}$-valued Wiener process with a finite trace nuclear covariance operator $Q \geq 0$ defined on a filtered complete probability space $\left(\Omega, \mathscr{F},\left\{\mathscr{F}_{t}\right\}_{t \geq 0}, P\right)$. The control function $u(\cdot)$ takes value in $L^{2}(J, \mathbb{U})$ of admissible control functions for a separable Hilbert space $\mathbb{U}$, and $B$ is a bounded linear operator from $\mathbb{U}$ into $\mathbb{M}$. The histories $x_{t}: \Omega \rightarrow C_{h}$ defined by $x_{t}=\{x(t+\theta), \theta \in(-\infty, 0]\}$ belong to the phase space $C_{h}$, which will be defined in Section 2. The initial data $\phi=$ $\{\phi(t), t \in(-\infty, 0]\}$ is an $\mathscr{F}_{0}$-measurable, $C_{h}$-valued random variable independent of $W$ with finite second moments, and $G: J \times C_{h} \rightarrow \mathbb{W}, \sigma: J \times J \times \mathbb{U} \rightarrow \mathscr{L}_{2}^{0}(\mathbb{K}, \mathbb{W})$ are appropriate mappings specified later (here, $\mathscr{L}_{2}^{0}(\mathbb{K}, \mathbb{H})$ denotes the space of all $Q$-Hilbert-Schmidt operators from $\mathbb{K}$ into $\mathbb{H}$, which is going to be defined later).

The structure of this paper is as follows. In Section 2, we briefly present some basic notations and preliminaries. The controllability result of system (1) is investigated by means of Sadovskii's fixed point theorem and operator theory in Section 3. Conclusion is given in Section 4.

\section{Preliminaries}

For more details in this section, we refer the reader to Pazy [26], Da Prato and Zabczyk [27], and Samko et al. [28]. Throughout this paper, $\left(\mathbb{M},|\cdot|_{\mathbb{Q}}\right)$ and $\left(\mathbb{K},\|\cdot\|_{\mathbb{K}}\right)$ denote two real separable Hilbert spaces. We denote by $\mathscr{L}(\mathbb{K}, \mathbb{H})$ the set of all linear bounded operators from $\mathbb{K}$ into $\mathbb{H}$, equipped with the usual operator norm $\|\cdot\|$. In this paper, we use the symbol $\|\cdot\|$ to denote norms of operators regardless of the spaces potentially involved when no confusion possibly arises.

Let $\left(\Omega, \mathscr{F},\left\{\mathscr{F}_{t}\right\}_{t \geq 0}, P\right)$ be a filtered complete probability space satisfying the usual condition, which means that the filtration is a right continuous increasing family and $\mathscr{F}_{0}$ contains all $P$-null sets. $W=\left(W_{t}\right)_{t \geq 0}$ is a $Q$-Wiener process defined on $\left(\Omega, \mathscr{F}_{,},\left\{\mathscr{F}_{t}\right\}_{t \geq 0}, P\right)$ with covariance operator $Q$ such that $\operatorname{Tr} Q<\infty$. We assume that there exist a complete orthonormal system $\left\{e_{k}\right\}_{k \geq 1}$ in $\mathbb{K}$, a bounded sequence of nonnegative real numbers $\lambda_{k}$ such that $Q e_{k}=\lambda_{k} e_{k}, k=$ $1,2, \ldots$, and a sequence of independent Brownian motions $\left\{\beta_{k}\right\}_{k \geq 1}$ such that

$$
(W(t), e)_{\mathbb{K}}=\sum_{k=1}^{\infty} \sqrt{\lambda_{k}}\left(e_{k}, e\right)_{\mathbb{K}} \beta_{k}(t), \quad e \in \mathbb{K}, t \geq 0 .
$$

Let $\mathscr{L}_{2}^{0}=\mathscr{L}_{2}\left(Q^{1 / 2} \mathbb{K}, \mathbb{H}\right)$ be the space of all Hilbert-Schmidt operators from $Q^{1 / 2} \mathbb{K}$ to $\mathbb{W}$ with the inner product $\langle\varphi, \phi\rangle_{\mathscr{L}_{2}^{0}}=$ $\operatorname{Tr}[\varphi Q \phi *]$.

Suppose that $0 \in \rho(-A)$, where $\rho(-A)$ is the resolvent set of $-A$, then the semigroup $S(\cdot)$ is uniformly bounded. That is to say, $\|S(t)\| \leq M, t \geq 0$, for some constant $M>0$. Then, for $\alpha \in(0,1]$, it is possible to define the fractional power operator $A^{\alpha}$ as a closed linear operator on its domain $\mathscr{D}\left(A^{\alpha}\right)$. Furthermore, the subspace $\mathscr{D}\left(A^{\alpha}\right)$ is dense in $\mathbb{H}$, and the expression

$$
\|x\|_{\alpha}=\left\|A^{\alpha} x\right\|, \quad x \in \mathscr{D}\left(A^{\alpha}\right)
$$

defines a norm on $\mathbb{H}_{\alpha}:=\mathscr{D}\left(A^{\alpha}\right)$. The following properties are well known.

Lemma 1 (Pazy [26]). Suppose that the preceding conditions are satisfied.

(a) If $0<\beta<\alpha \leq 1$, then $\mathbb{H}_{\alpha} \subset \mathbb{M}_{\beta}$ and the embedding is compact whenever the resolvent operator of $A$ is compact.

(b) For every $\alpha \in(0,1]$, there exists a positive constant $C_{\alpha}$ such that

$$
\left\|A^{\alpha} S(t)\right\| \leq \frac{C_{\alpha}}{t^{\alpha}}, \quad t>0
$$

Let now us recall some basic definitions and results of fractional calculus.

Definition 2. The fractional integral of order $\alpha$ with the lower limit 0 for a function $f$ is defined as

$$
I^{\alpha} f=\frac{1}{\Gamma(\alpha)} \int_{0}^{t} \frac{f(s)}{(t-s)^{1-\alpha}} d s, \quad t>0, \alpha>0,
$$

provided the right-hand side is pointwise defined on $[0, \infty)$, where $\Gamma(\cdot)$ is the gamma function.

Definition 3. The Caputo derivative of order $\alpha$ with the lower limit 0 for a function $f$ can be written as

$$
\begin{aligned}
{ }^{c} D^{\alpha} f(t) & =\frac{1}{\Gamma(n-\alpha)} \int_{0}^{t} \frac{f^{n}(s)}{(t-s)^{\alpha+1-n}} d s \\
& =I^{n-\alpha} f^{n}(t), \quad t>0,0 \leq n-1<\alpha<n .
\end{aligned}
$$

If $f$ is an abstract function with values in $\mathbb{H}$, then the integrals that appear in the previous definitions are taken in Bochner's sense. 
Assume that $h:(-\infty, 0] \rightarrow(0,+\infty)$ with $l=\int_{-\infty}^{0} h(t) d t<$ $+\infty$ is a continuous function. Recall that the abstract phase space $\mathscr{C}_{h}$ is defined by

$$
\mathscr{C}_{h}=\left\{\varphi:(-\infty, 0] \longrightarrow \mathbb{H}, \text { for any } a>0,\left(E|\varphi(\theta)|^{2}\right)^{1 / 2}\right.
$$

is abounded and measurable function on $[-a, 0]$,

$$
\left.\int_{-\infty}^{0} h(s) \sup _{s \leq \theta \leq 0}\left(E|\varphi(\theta)|^{2}\right)^{1 / 2} d s<+\infty\right\} .
$$

If $\mathscr{C}_{h}$ is endowed with the norm

$$
\|\varphi\|_{\mathscr{C}_{h}}=\int_{-\infty}^{0} h(s) \sup _{s \leq \theta \leq 0}\left(E|\varphi(\theta)|^{2}\right)^{1 / 2} d s, \quad \varphi \in \mathscr{C}_{h}
$$

then $\left(\mathscr{C}_{h},\|\cdot\|_{\mathscr{C}_{h}}\right)$ is a Banach space (see Liu et al. [29]).

At the end of this section, we recall the fixed point theorem of Sadovskii [30].

Lemma 4. Let $\Phi$ be a condensing operator on a Banach space $\mathbb{H}$; that is, $\Phi$ is continuous, and take bounded sets into bounded sets, and $\mu(\Phi(B)) \leq \mu(B)$ for every bounded set $B$ of $\mathbb{M}$ with $\mu(B)>0$. If $\Phi(N) \subset N$ for a convex, closed, and bounded set $N$ of $\mathbb{H}$, then $\Phi$ has a fixed point in $\mathbb{H}$ (where $\mu(\cdot)$ denotes Kuratowski's measure of noncompactness.)

\section{Main Results}

In this section, we obtain controllability of system (1). We first present the definition of mild solutions.

Definition 5. An $\mathbb{H}$-valued stochastic process $\{x(t), t \in$ $(-\infty, b]\}$ is said to be a mild solution of system (1) if

(i) $x(t)$ is $\mathscr{F}_{t}$-adapted and measurable for each $t \geq 0$;

(ii) $x(t)$ is continuous on $[0, b]$ almost surely and for each $s \in[0, t)$, the function $(t-s)^{\alpha-1} A T_{\alpha}(t-s) G\left(s, x_{s}\right)$ is integrable such that the following stochastic integral equation is verified:

$$
\begin{aligned}
x(t)= & S_{\alpha}(t)[\phi(0)+G(0, \phi)]-G\left(t, x_{t}\right) \\
& -\int_{0}^{t}(t-s)^{\alpha-1} A T_{\alpha}(t-s) G\left(s, x_{s}\right) d s \\
& +\int_{0}^{t}(t-s)^{\alpha-1} T_{\alpha}(t-s) B u(s) d s \\
& +\int_{0}^{t}(t-s)^{\alpha-1} T_{\alpha}(t-s) \\
& \times\left[\int_{0}^{s} \sigma\left(s, \tau, x_{\tau}\right) d W(\tau)\right] d s ;
\end{aligned}
$$

(iii) $x(t)=\phi(t)$ on $(-\infty, 0]$ with $\|\phi\|_{\mathscr{C}_{h}}^{2}<\infty$,

where

$$
\begin{gathered}
S_{\alpha}(t) x=\int_{0}^{\infty} \eta_{\alpha}(\theta) S\left(t^{\alpha} \theta\right) x d \theta, \\
T_{\alpha}(t) x=\alpha \int_{0}^{\infty} \theta \eta_{\alpha}(\theta) S\left(t^{\alpha} \theta\right) x d \theta
\end{gathered}
$$

with $\eta_{\alpha}$ a probability density function defined on $(0, \infty)$.

Definition 6. System (1) is said to be controllable on the interval $J$, if for every initial stochastic process $\phi \in \mathscr{C}_{h}$ defined on $(-\infty, 0]$, there exists a stochastic control $u \in$ $L^{2}(J, \mathbb{U})$, which is adapted to the filtration $\left\{\mathscr{F}_{t}\right\}_{t \geq 0}$ such that the mild solution $x(t)$ of (1) satisfies $x(b)=x^{*}$, where $x^{*}$ and $b$ are preassigned terminal state and time, respectively.

The following properties of $S_{\alpha}(t)$ and $T_{\alpha}(t)$ that appeared in Zhou and Jiao [7] are useful.

Lemma 7. Under previous assumptions on $S(t), t \geq 0$, and $A$, then

(i) for any fixed $t \geq 0, S_{\alpha}(t)$ and $T_{\alpha}(t)$ are linear and bounded operators such that for any $x \in \mathbb{U},\left|S_{\alpha}(t) x\right|_{\mathbb{Q}} \leq$ $M|x|_{\mathbb{\square}},\left|T_{\alpha}(t) x\right|_{\mathbb{\sharp}} \leq(M \alpha / \Gamma(1+\alpha))|x|_{\mathbb{\sharp}} ;$

(ii) $S_{\alpha}(t)$ and $T_{\alpha}(t)$ are strongly continuous;

(iii) for any $x \in \mathbb{H}, \beta \in(0,1)$, and $\theta \in(0,1]$, one has

$$
\begin{gathered}
A T_{\alpha}(t) x=A^{1-\beta} T_{\alpha}(t) A^{\beta} x, \\
\left\|A^{\theta} T_{\alpha}(t)\right\| \leq \frac{\alpha C_{\theta}}{t^{\alpha \theta}} \frac{\Gamma(2-\theta)}{\Gamma(1+\alpha(1-\theta))}, \quad t \in[0, b] .
\end{gathered}
$$
tions.

In this paper, we will work under the following assump-

$\left(\mathrm{A}_{0}\right)-A$ is the infinitesimal generator of an analytic semigroup of bounded linear operators $S(t)$ in $\mathbb{H}$; $S(t)$, $t>0$, is continuous in the uniform operator topology and $\|S(t)\| \leq M$ for some $M>0$.

$\left(\mathrm{A}_{1}\right)$ The function $G: J \times \mathscr{C}_{h} \rightarrow \mathbb{H}$ is continuous, and there exist some constants $M_{G}>0, \beta \in(0,1)$ with $\alpha \beta>1 / 2$, such that $G$ is $\mathbb{H}_{\beta}$-valued and

$$
\begin{aligned}
& E\left|A^{\beta} G(t, x)-A^{\beta} G(t, y)\right|_{\mathbb{\square}}^{2} \\
& \quad \leq M_{G}\|x-y\|_{\mathscr{C}_{h}}^{2}, \quad x, y \in \mathscr{C}_{h}, t \in J, \\
& E\left|A^{\beta} G(t, x)\right|_{\mathbb{\square}}^{2} \leq M_{G}\left(\|x\|_{\mathscr{C}_{h}}^{2}+1\right) .
\end{aligned}
$$

$\left(\mathrm{A}_{2}\right) \sigma: J \times J \times \mathscr{C}_{h} \rightarrow L(\mathbb{K}, \mathbb{M})$ satisfies the following:

(2a) for each $(t, s) \in D:=J \times J, \sigma(t, s, \cdot): \mathscr{C}_{h} \rightarrow$ $L(\mathbb{K}, \mathbb{H})$ is continuous and for each $x \in \mathscr{C}_{h}$, $\sigma(\cdot, \cdot, x): D \rightarrow L(\mathbb{K}, \mathbb{H})$ is strongly measurable; 
(2b) there is a positive integrable function $m \in$ $L^{1}([0, b])$ and a continuous nondecreasing function $\Xi:[0, \infty) \rightarrow(0, \infty)$ such that for every $(t, s, x) \in J \times J \times \mathscr{C}_{h}$, we have

$$
\begin{gathered}
\int_{0}^{t} E|\sigma(t, s, x)|_{\mathscr{L}_{2}^{0}}^{2} d s \leq m(t) \Xi\left(\|x\|_{\mathscr{C}_{h}}^{2}\right), \\
\liminf _{r \rightarrow \infty} \frac{\Xi(r)}{r} d s=\Lambda<\infty ;
\end{gathered}
$$

(2c) for any $x, y \in \mathscr{C}_{h}, t \geq 0$, there exists a positive constant $M_{\sigma}$ such that

$$
\begin{gathered}
\int_{0}^{t} E|\sigma(t, s, x)-\sigma(t, s, y)|_{\mathscr{L}_{2}^{0}}^{2} d s \\
\leq M_{\sigma}\|x-y\|_{\mathscr{C}_{h}}^{2} .
\end{gathered}
$$

$\left(\mathrm{A}_{3}\right)$ The linear operator $W$ from $L^{2}(J, \mathbb{U})$ into $\mathbb{U}$ defined by

$$
W u=\int_{0}^{b}(b-s)^{\alpha-1} T_{\alpha}(b-s) B u(s) d s
$$

has an invertible operator $W^{-1}$ defined on $L^{2}(J, \mathbb{U}) /$ ker $W$ (see [31]), and there exist a pair of constants $N_{1}, N_{2}>0$ such that

$$
\|B\|^{2} \leq N_{1}, \quad\left\|W^{-1}\right\| \leq N_{2} .
$$

$\left(\mathrm{A}_{4}\right)$ There exists a compact set $\mho \subseteq \mathbb{U}$ such that $S(t) \sigma(s, \tau, \xi) \in \mho$ for all $t \in J, 0 \leq \tau \leq s \leq b$ and $\xi \in \mathscr{C}_{h}$ with finite second moment.

$\left(\mathrm{A}_{5}\right)$ Assume that the following relationship holds:

$$
\begin{aligned}
20(1 & \left.+5 N_{1} N_{2} N\right) l^{2} \\
& \times\left(M_{0}+M_{G} P+N b^{-1} \operatorname{Tr} Q \Lambda \int_{0}^{b} m(s) d s\right)<1,
\end{aligned}
$$

where

$$
\begin{gathered}
N=\frac{b^{2 \alpha}}{2 \alpha-1}\left(\frac{M \alpha}{\Gamma(1+\alpha)}\right)^{2}, \quad M_{0}=\left\|A^{-\beta}\right\|^{2} M_{G}, \\
P=\frac{\alpha^{2} C_{1-\beta}^{2} \Gamma^{2}(1+\beta)}{\Gamma^{2}(1+\alpha \beta)} \frac{b^{2 \alpha \beta}}{2 \alpha \beta-1} .
\end{gathered}
$$

Denote by $C((-\infty, b], \mathbb{U})$ the space of all continuous $\mathbb{H}$ valued stochastic processes $\{\xi(t), t \in(-\infty, b]\}$. Let

$$
\mathscr{C}_{b}=\left\{x: x \in C((-\infty, b], \mathbb{U}), x_{0}=\phi \in \mathscr{C}_{h}\right\} .
$$

Set $\|\cdot\|_{b}$ to be a seminorm defined by

$$
\|x\|_{b}=\left\|x_{0}\right\|_{\mathscr{C}_{h}}+\sup _{s \in[0, b]}\left(E|x(s)|^{2}\right)^{1 / 2}, \quad x \in \mathscr{C}_{b} .
$$

We have the following useful lemma that appeared in Liu et al. [29].
Lemma 8. Assume that $x \in \mathscr{C}_{b}$; then, for all $t \in J, x_{t} \in \mathscr{C}_{h}$. Moreover,

$$
l\left(E|x(t)|^{2}\right)^{1 / 2} \leq\left\|x_{t}\right\|_{\mathscr{C}_{h}} \leq l \sup _{s \in[0, t]}\left(E|x(s)|^{2}\right)^{1 / 2}+\left\|x_{0}\right\|_{\mathscr{C}_{h}},
$$

where $l=\int_{-\infty}^{0} h(s) d s<\infty$.

The main object of this paper is to explain and prove the following theorem.

Theorem 9. Assume that assumptions $\left(A_{0}\right)-\left(A_{5}\right)$ hold. Then, system (1) is controllable on $J$ provided that

$$
\begin{aligned}
L:= & 3 l^{2}\left(1+3 N_{1} N_{2} N\right)\left(M_{0}+M_{G} P\right) \\
& +9 N_{1} N_{2} N^{2} \operatorname{Tr} Q M_{\sigma} l^{2}<1 .
\end{aligned}
$$

Proof. Using assumption $\left(\mathrm{A}_{3}\right)$, for an arbitrary process $x(\cdot)$, define the control process

$$
\begin{array}{r}
u_{x}(t)=W^{-1}\left\{x^{*}-S_{\alpha}(b)[\phi(0)+G(0, \phi)]+G\left(b, x_{b}\right)\right. \\
\quad+\int_{0}^{b}(b-s)^{\alpha-1} A T_{\alpha}(b-s) G\left(s, x_{s}\right) d s \\
\quad-\int_{0}^{b}(b-s)^{\alpha-1} T_{\alpha}(b-s) \\
\left.\quad \times\left[\int_{0}^{s} \sigma\left(s, \tau, x_{\tau}\right) d W(\tau)\right] d s\right\}(t) .
\end{array}
$$

We transform (1) into a fixed point problem. Consider the operator $Q: \mathscr{C}_{b} \rightarrow \mathscr{C}_{b}$ defined by

$(Q x)(t)$

$$
=\left\{\begin{array}{lr}
\phi(t), & t \in(-\infty, 0] ; \\
S_{\alpha}(t)[\phi(0)+G(0, \phi)]-G\left(t, x_{t}\right) & \\
\quad-\int_{0}^{t}(t-s)^{\alpha-1} A T_{\alpha}(t-s) G\left(s, x_{s}\right) d s & \\
+\int_{0}^{t}(t-s)^{\alpha-1} T_{\alpha}(t-s) B u_{x}(s) d s & \\
+\int_{0}^{t}(t-s)^{\alpha-1} T_{\alpha}(t-s) & \\
& \times\left[\int_{0}^{s} \sigma\left(s, \tau, x_{\tau}\right) d W(\tau)\right] d s, \quad t \in J .
\end{array}\right.
$$

It follows from Hölder inequality, Lemma 7, and assumption $\left(\mathrm{A}_{1}\right)$ that

$$
\begin{gathered}
E\left|\int_{0}^{t}(t-s)^{\alpha-1} A T_{\alpha}(t-s) G\left(s, x_{s}\right) d s\right|_{\mathbb{H}}^{2} \\
\leq E\left[\int_{0}^{t} \mid(t-s)^{\alpha-1} A^{1-\beta} T_{\alpha}\right. \\
\left.\times\left.(t-s) A^{\beta} G\left(s, x_{s}\right)\right|_{\mathbb{H}} d s\right]^{2}
\end{gathered}
$$




$$
\begin{aligned}
& \leq P E\left[\int_{0}^{t} \mid(t-s)^{\alpha(\beta-1)}(t-s)^{\alpha-1}\right. \\
& \left.\quad \times\left. A^{\beta} G\left(s, x_{s}\right)\right|_{\mathbb{G}-1} d s\right]^{2} \\
& \leq P \int_{0}^{t}(t-s)^{2(\alpha \beta-1)} d s \\
& \quad \times \int_{0}^{t} M_{G}\left(1+\left|x_{s}\right|_{\mathscr{C}_{h}}^{2}\right) d s,
\end{aligned}
$$

which deduces that $(t-s)^{\alpha-1} A T_{\alpha}(t-s) G\left(s, x_{s}\right)$ is integrable on $J$ by Bochner's theorem (see [32]), where $P$ is defined in (19).

In what follows, we will show that using the control $u_{x}(\cdot)$, the operator $Q$ has a fixed point, which is then a mild solution for system (1).

For $\phi \in \mathscr{C}_{h}$, define

$$
\widetilde{\phi}(t)= \begin{cases}\phi(t), & t \in(-\infty, 0] \\ S_{\alpha}(t) \phi(0), & t \in J\end{cases}
$$

Then, $\widetilde{\phi} \in \mathscr{C}_{b}$. Set $x(t)=\widetilde{\phi}(t)+z(t), t \in(-\infty, b]$. It is easy to check that $x$ satisfies (1) if and only if $z_{0}=0$ and

$$
\begin{aligned}
z(t)= & S_{\alpha}(t) G(0, \phi)-G\left(t, \tilde{\phi}_{t}+z_{t}\right) \\
& -\int_{0}^{t}(t-s)^{\alpha-1} A T_{\alpha}(t-s) G\left(s, \tilde{\phi}_{s}+z_{s}\right) d s \\
& +\int_{0}^{t}(t-s)^{\alpha-1} T_{\alpha}(t-s) B u_{\tilde{\phi}+z}(s) d s \\
& +\int_{0}^{t}(t-s)^{\alpha-1} T_{\alpha}(t-s) \\
& \quad \times\left[\int_{0}^{s} \sigma\left(s, \tau, \tilde{\phi}_{\tau}+z_{\tau}\right) d W(\tau)\right] d s,
\end{aligned}
$$

where $u_{\widetilde{\phi}+z}$ is obtained by replacing $x_{t}$ by $\widetilde{\phi}_{t}+z_{t}$ in (24). Let

$$
\mathscr{C}_{b}^{0}=\left\{z \in \mathscr{C}_{b}, z_{0}=0 \in \mathscr{C}_{h}\right\}
$$

For each $z \in \mathscr{C}_{b}^{0}$, we have

$$
\|z\|_{b}=\left\|z_{0}\right\|_{\mathscr{C}_{h}}+\sup _{s \in[0, b]}\left(E|z(s)|^{2}\right)^{1 / 2}=\sup _{s \in[0, b]}\left(E|z(s)|^{2}\right)^{1 / 2} .
$$

Thus, $\left(\mathscr{C}_{b}^{0},\|\cdot\|_{b}\right)$ is a Banach space. For $q>0$, set

$$
B_{q}=\left\{y \in \mathscr{C}_{b}^{0},\|y\|_{b}^{2} \leq q\right\}
$$

then, for each $q, B_{q}$ is clearly a bounded closed convex set in $\mathscr{C}_{b}^{0}$. For $z \in B_{q}$, from Lemma 8 , we see that

$$
\begin{aligned}
&\left\|z_{t}+\widetilde{\phi}_{t}\right\|_{\mathscr{C}_{h}}^{2} \leq 2\left(\left\|z_{t}\right\|_{\mathscr{C}_{h}}^{2}+\left\|\widetilde{\phi}_{t}\right\|_{\mathscr{C}_{h}}^{2}\right) \\
& \leq 4\left(l^{2} \sup _{s \in[0, t]} E|z(s)|^{2}+\left\|z_{0}\right\|_{\mathscr{C}_{h}}^{2}\right. \\
&\left.\quad l^{2} \sup _{s \in[0, t]} E|\widetilde{\phi}(s)|^{2}+\left\|\widetilde{\phi}_{0}\right\|_{\mathscr{C}_{h}}^{2}\right) \\
& \leq 4 l^{2}\left(q+M^{2} E|\phi(0)|_{\mathbb{G}}^{2}\right)+4\|\phi\|_{\mathscr{C}_{h}}^{2} .
\end{aligned}
$$

Consider the map $\Pi: \mathscr{C}_{b}^{0} \rightarrow \mathscr{C}_{b}^{0}$ defined by

$(\Pi z)(t)$

$$
\begin{cases}0, & t \in(-\infty, 0] ; \\ S_{\alpha}(t) G(0, \phi)-G\left(t, \widetilde{\phi}_{t}+z_{t}\right) & \\ & -\int_{0}^{t}(t-s)^{\alpha-1} A T_{\alpha}(t-s) \\ & \times G\left(s, \widetilde{\phi}_{s}+z_{s}\right) d s \\ & +\int_{0}^{t}(t-s)^{\alpha-1} T_{\alpha}(t-s) B u_{\tilde{\phi}+z}(s) d s \\ & +\int_{0}^{t}(t-s)^{\alpha-1} T_{\alpha}(t-s) \\ & \times\left[\int_{0}^{s} \sigma\left(s, \tau, \tilde{\phi}_{\tau}+z_{\tau}\right) d W(\tau)\right] d s, \quad t \in J .\end{cases}
$$

A similar argument as (26), we can show that $\Pi$ is well defined on $B_{q}$ for each $q>0$. Note that the operator $Q$ with a fixed point is equivalent to show that the operator $\Pi$ has fixed point. To this end, we decompose $\Pi$ as $\Pi=\Pi_{1}+\Pi_{2}$, where the operators $\Pi_{1}$ and $\Pi_{2}$ are defined on $B_{q}$, respectively, by

$$
\begin{aligned}
\left(\Pi_{1} z\right)(t)= & S_{\alpha}(t) G(0, \phi)-G\left(t, \tilde{\phi}_{t}+z_{t}\right) \\
& \quad-\int_{0}^{t}(t-s)^{\alpha-1} A T_{\alpha}(t-s) G\left(s, \widetilde{\phi}_{s}+z_{s}\right) d s \\
& +\int_{0}^{t}(t-s)^{\alpha-1} T_{\alpha}(t-s) B u_{\tilde{\phi}+z}(s) d s, \\
\left(\Pi_{2} z\right)(t)= & \int_{0}^{t}(t-s)^{\alpha-1} T_{\alpha}(t-s) \\
& \times\left[\int_{0}^{s} \sigma\left(s, \tau, \widetilde{\phi}_{\tau}+z_{\tau}\right) d W(\tau)\right] d s .
\end{aligned}
$$

Thus, Theorem 9 follows from the next theorem.

Theorem 10. Assume that the assumptions of Theorem 9 hold. Then, $\Pi=\Pi_{1}+\Pi_{2}$ satisfies all the conditions in Lemma 4.

Proof. The proof is followed by the several steps.

Step 1 . There exists a positive number $q$ such that $\Pi\left(B_{q}\right) \subset B_{q}$. If it is not true, then for each positive number $q$, there exists a 
function $z^{q}(\cdot) \in B_{q}$, but $\Pi\left(z^{q}\right) \notin B_{q}$; that is, $E\left|\left(\Pi z^{q}\right)(t)\right|_{\varpi \mathbb{N}}^{2}>q$ for some $t=t(q) \in J$. An elementary calculation can show that

$$
\begin{aligned}
& q \leq E\left|\Pi\left(z^{q}\right)(t)\right|_{\mathbb{H}}^{2} \\
& \leq 5 E\left|S_{\alpha}(t) G(0, \phi)\right|_{\mathscr{H}}^{2}+5 E\left|G\left(t, \tilde{\phi}_{t}+z_{t}^{q}\right)\right|_{\mathbb{H}}^{2} \\
& +5 E\left|\int_{0}^{t}(t-s)^{\alpha-1} A T_{\alpha}(t-s) G\left(s, \widetilde{\phi}_{s}+z_{s}^{q}\right) d s\right|_{\oplus \rightarrow}^{2} \\
& +5 E \mid \int_{0}^{t}(t-s)^{\alpha-1} T_{\alpha}(t-s) \\
& \times\left.\left[\int_{0}^{s} \sigma\left(s, \widetilde{\phi}_{\tau}+z_{\tau}^{q}\right) d W(\tau)\right] d s\right|_{\mathscr{H}} ^{2} \\
& +5 E\left|\int_{0}^{t}(t-s)^{\alpha-1} T_{\alpha}(t-s) B u_{\tilde{\phi}+z^{q}}(s) d s\right|_{\mathbb{H}}^{2} \\
& =5 \sum_{i=1}^{5} I_{i} \text {. }
\end{aligned}
$$

We estimate each term on the right hand side of (35). By virtue of Lemma 8 , assumptions $\left(A_{0}\right)-\left(A_{1}\right)$, and (32), we obtain

$$
\begin{aligned}
I_{1}+ & I_{2} \\
\leq & M^{2}\left\|A^{-\beta}\right\|^{2} E\left|A^{\beta} G(0, \phi)\right|_{\mathbb{H}}^{2} \\
& +\left\|A^{-\beta}\right\|^{2} E\left|A^{\beta} G\left(t, \widetilde{\phi}_{t}+z_{t}^{q}\right)\right|_{\mathbb{H}}^{2} \\
\leq & M_{0}\left[M^{2}\left(1+\|\phi\|_{\mathscr{C}_{h}}^{2}\right)+\left(1+\left\|\tilde{\phi}_{t}+z_{t}^{q}\right\|_{\mathscr{C}_{h}}^{2}\right)\right] \\
\leq & M_{0}\left[M^{2}\left(1+\|\phi\|_{\mathscr{C}_{h}}^{2}\right)\right. \\
& \left.\quad+\left(1+4 l^{2}\left(q+M^{2} E|\phi(0)|_{\mathbb{H}}^{2}\right)+4\|\phi\|_{\mathscr{C}_{h}}^{2}\right)\right] ;
\end{aligned}
$$

in view of Lemma 7 and Hölder inequality, we have

$$
\begin{aligned}
& I_{3} \leq E\left[\int_{0}^{t} \mid(t-s)^{\alpha-1} A^{1-\beta} T_{\alpha}(t-s)\right. \\
&\left.\times\left. A^{\beta} G\left(s, \widetilde{\phi}_{s}+z_{s}^{q}\right)\right|_{\mathbb{H}} d s\right]^{2} \\
& \leq \frac{\alpha^{2} C_{1-\beta}^{2} \Gamma^{2}(1+\beta)}{\Gamma^{2}(1+\alpha \beta)} E \\
& \quad \times\left[\int_{0}^{t}\left|(t-s)^{\alpha \beta-1} A^{\beta} G\left(s, \widetilde{\phi}_{s}+z_{s}^{q}\right)\right|_{\mathbb{H}} d s\right]^{2}
\end{aligned}
$$

$$
\begin{aligned}
\leq & \frac{\alpha^{2} C_{1-\beta}^{2} \Gamma^{2}(1+\beta)}{\Gamma^{2}(1+\alpha \beta)} \int_{0}^{t}(t-s)^{2(\alpha \beta-1)} d s \\
& \times \int_{0}^{t} M_{G}\left(1+\left|\widetilde{\phi}_{s}+z_{s}^{q}\right|_{\mathscr{C}_{h}}^{2}\right) d s \\
\leq & \frac{M_{G} \alpha^{2} C_{1-\beta}^{2} \Gamma^{2}(1+\beta)}{\Gamma^{2}(1+\alpha \beta)} \frac{b^{2 \alpha \beta-1}}{2 \alpha \beta-1} \\
& \times \int_{0}^{t}\left(1+\left|\widetilde{\phi}_{s}+z_{s}^{q}\right|_{\mathscr{C}_{h}}^{2}\right) d s \\
\leq & M_{G} P\left(1+4 l^{2}\left(q+M^{2} E|\phi(0)|_{\mathbb{⿴}}^{2}\right)+4\|\phi\|_{\mathscr{C}_{h}}^{2}\right),
\end{aligned}
$$

where $M_{0}$ and $P$ are defined in (18) and (19), respectively.

Applying Burkhölder-Davis-Gundy's inequality and assumptions $\left(\mathrm{A}_{2}\right)$, we get

$$
\begin{aligned}
I_{4} \leq & E\left[\int_{0}^{t} \mid(t-s)^{\alpha-1} T_{\alpha}(t-s)\right. \\
& \left.\times\left.\left[\int_{0}^{s} \sigma\left(s, \tau, \widetilde{\phi}_{\tau}+z_{\tau}^{q}\right) d W(\tau)\right]\right|_{\uplus} d s\right]^{2} \\
\leq & \left(\frac{M \alpha}{\Gamma(1+\alpha)}\right)^{2} \int_{0}^{t}(t-s)^{2(\alpha-1)} d s \\
& \times \int_{0}^{t} E\left|\int_{0}^{s} \sigma\left(s, \tau, \widetilde{\phi}_{\tau}+z_{\tau}^{q}\right) d W(\tau)\right|_{\bullet}^{2} d s \\
\leq & \left(\frac{M \alpha}{\Gamma(1+\alpha)}\right)^{2} \frac{b^{2 \alpha-1}}{2 \alpha-1} \\
& \times \int_{0}^{t} \operatorname{Tr} Q \int_{0}^{s} E\left|\sigma\left(s, \widetilde{\phi}_{\tau}+z_{\tau}^{q}\right)\right|_{\mathscr{L}_{2}^{0}}^{2} d \tau d s \\
\leq & \left(\frac{M \alpha}{\Gamma(1+\alpha)}\right)^{2} \frac{b^{2 \alpha-1}}{2 \alpha-1} \operatorname{Tr} Q \\
& \times \int_{0}^{t} m(s) \Xi\left(\left\|\widetilde{\phi}_{\tau}+z_{\tau}^{q}\right\|_{\mathscr{C}_{h}}^{2}\right) d s \\
\leq & \left(\frac{M \alpha}{\Gamma(1+\alpha)}\right)^{2} \frac{\operatorname{Tr} Q b^{2 \alpha-1}}{2 \alpha-1} \Xi \\
& \times\left(4 l^{2}\left(q+M^{2} E|\phi(0)|_{\sharp \downarrow}^{2}\right)+4\|\phi\|_{\mathscr{C}_{h}}^{2}\right) \int_{0}^{t} m(s) d s .
\end{aligned}
$$

On the other hand, in view of $(24)$ and $\left(\mathrm{A}_{3}\right)$, we have

$$
\begin{aligned}
E\left|B u_{z^{q}+\tilde{\phi}}(t)\right|_{[-1}^{2} & \\
\leq N_{1} N_{2} E \mid\{ & x^{*}-S_{\alpha}(b)[\phi(0)+G(0, \phi)] \\
& +G\left(b, \widetilde{\phi}_{b}+z_{b}^{q}\right) \\
& +\int_{0}^{b}(b-s)^{\alpha-1} A T_{\alpha}(b-s) G\left(s, \widetilde{\phi}_{s}+z_{s}^{q}\right) d s
\end{aligned}
$$




$$
\begin{aligned}
& -\int_{0}^{b}(b-s)^{\alpha-1} T_{\alpha}(b-s) \\
& \left.\quad \times\left[\int_{0}^{s} \sigma\left(s, \tau, \tilde{\phi}_{\tau}+z_{\tau}^{q}\right) d W(\tau)\right] d s\right\}\left.(t)\right|_{\mathbb{\boxplus}} ^{2} ;
\end{aligned}
$$

thus, by the same procedure as (36)-(38), it follows that

$$
\begin{aligned}
I_{5} \leq\left(\frac{M \alpha}{\Gamma(1+\alpha)}\right)^{2} \int_{0}^{t}(t-s)^{2(\alpha-1)} d s \int_{0}^{t} E\left|B u_{z^{q}+\tilde{\phi}}(s)\right|_{\mathbb{\square}}^{2} d s \\
\leq N\left\{C_{0}+5 N_{1} N_{2}\right. \\
\times\left[4 M_{G}\left\|A^{-\beta}\right\|^{2} l^{2} q+4 l^{2} M_{G} P q\right. \\
+2 N b^{-1} \operatorname{Tr} Q \Xi \\
\times\left(4 l^{2}\left(q+M^{2} E|\phi(0)|_{\mathbb{G}}^{2}\right)+4\|\phi\|_{\mathscr{C}_{h}}^{2}\right) \\
\left.\left.\times \int_{0}^{b} m(s) d s\right]\right\},
\end{aligned}
$$

where

$$
\begin{aligned}
C_{0}=5 N_{1} N_{2}[ & E\left|x^{*}\right|_{\mathbb{G}}^{2}+2 M^{2} E|\phi(0)|_{\mathbb{G}}^{2} \\
& +2 M^{2} M_{0}\left(1+\|\phi\|_{\mathscr{C}_{h}}^{2}\right) \\
& +M_{0}\left(1+4 l^{2} M^{2} E|\phi(0)|_{\mathbb{G}}^{2}+4\|\phi\|_{\mathscr{C}_{h}}^{2}\right) \\
& \left.+M_{G} P\left(1+4 l^{2} M^{2} E|\phi(0)|_{\mathbb{G}}^{2}+4\|\phi\|_{\mathscr{C}_{h}}^{2}\right)\right] .
\end{aligned}
$$

$M_{0}$ and $P$ are defined in (18) and (19), respectively.

Combining these estimates (35) to (40) yields

$$
\begin{aligned}
q \leq & E\left|\Pi\left(z^{q}\right)(t)\right|_{\mathbb{q}}^{2} \\
\leq & L_{0}+20 M_{0} l^{2}\left(1+5 N_{1} N_{2} N\right) q \\
& +20 l^{2} M_{G} P\left(1+5 N_{1} N_{2} N\right) q \\
& +5\left(\frac{M \alpha}{\Gamma(1+\alpha)}\right)^{2} \frac{\operatorname{Tr} Q b^{2 \alpha-1}}{2 \alpha-1} \\
& \times N \Xi\left(4 l^{2}\left(q+M^{2} E|\phi(0)|_{\mathbb{G}}^{2}\right)+4\|\phi\|_{\mathscr{C}_{h}}^{2}\right) \int_{0}^{b} m(s) d s,
\end{aligned}
$$

where

$$
\begin{aligned}
L_{0}= & 5 N+5 M^{2} M_{0}\left(1+\|\phi\|_{\mathscr{C}_{h}}^{2}\right) C_{0} \\
& +5 M_{0}\left(1+4 l^{2} M^{2} E|\phi(0)|_{\mathbb{G}}^{2}+4\|\phi\|_{\mathscr{C}_{h}}^{2}\right) \\
& +5 M_{G} P\left(1+4 l^{2} M^{2} E|\phi(0)|_{\mathbb{\square}}^{2}+4\|\phi\|_{\mathscr{C}_{h}}^{2}\right) .
\end{aligned}
$$

Dividing both sides of (42) by $q$ and taking $q \rightarrow \infty$, we obtain that

$$
\begin{aligned}
20(1 & \left.+5 N_{1} N_{2} N\right) l^{2} \\
& \times\left(M_{0}+M_{G} P+N b^{-1} \operatorname{Tr} Q \Lambda \int_{0}^{b} m(s) d s\right) \geq 1,
\end{aligned}
$$

which is a contradiction by assumption $\left(\mathrm{A}_{5}\right)$. Thus, for some positive number $q, \Pi\left(B_{q}\right) \subset B_{q}$.

Step 2. $\Pi_{1}$ is a contractive mapping. Let $x, v \in B_{q}$. From the assumptions on $G$ and $\sigma$, it is easy to verify that the following inequality holds:

$$
\begin{aligned}
E\left|u_{x+\tilde{\phi}}(s)-u_{v+\tilde{\phi}}(s)\right|_{\mathbb{H}}^{2} & \\
\leq 3 N_{2}\left[\left\|A^{-\beta}\right\|^{2} M_{G}\left\|x_{b}-v_{b}\right\|_{\mathscr{C}_{h}}^{2}\right. & \\
& +\frac{\alpha^{2} C_{1-\beta}^{2} \Gamma^{2}(1+\beta)}{\Gamma^{2}(1+\alpha \beta)} \frac{b^{2 \alpha \beta-1}}{2 \alpha \beta-1} \int_{0}^{b} M_{G}\left\|x_{s}-v_{s}\right\|_{\mathscr{C}_{h}}^{2} d s \\
& \left.+\left(\frac{M \alpha}{\Gamma(1+\alpha)}\right)^{2} \frac{b^{2 \alpha-1}}{2 \alpha-1} \int_{0}^{b} M_{\sigma} \operatorname{Tr} Q\left\|x_{s}-v_{s}\right\|_{\mathscr{C}_{h}}^{2} d s\right] .
\end{aligned}
$$

Thus, by the assumptions, we have

$$
\begin{aligned}
& E\left|\left(\Pi_{1} x\right)(t)-\left(\Pi_{1} v\right)(t)\right|_{\mathbb{\square}}^{2} \\
& \leq 3 E\left|G\left(t, \tilde{\phi}_{t}+x_{t}\right)-G\left(t, \tilde{\phi}_{t}+v_{t}\right)\right|_{\mathbb{q}}^{2} \\
& +3 E \mid \int_{0}^{t}(t-s)^{\alpha-1} A T_{\alpha}(t-s) \\
& \times\left.\left[G\left(s, \tilde{\phi}_{s}+x_{s}\right)-G\left(s, \tilde{\phi}_{s}+v_{s}\right)\right] d s\right|_{\mathbb{U}} ^{2} \\
& +3 E\left|\int_{0}^{t}(t-s)^{\alpha-1} T_{\alpha}(t-s) B\left(u_{\tilde{\phi}+x}-u_{\tilde{\phi}+v}\right) d s\right|_{\mathbb{Q}}^{2} \\
& \leq 3 M_{0} l^{2}\left(1+3 N_{1} N_{2} N\right) \sup _{0 \leq s \leq b} E|x(s)-v(s)|_{\mathbb{Q}}^{2} \\
& +3 P M_{G} l^{2}\left(1+3 N_{1} N_{2} N\right) \sup _{0 \leq s \leq b} E|x(s)-v(s)|_{\mathbb{q}}^{2} \\
& +9 N_{1} N_{2} N^{2} \operatorname{Tr} Q M_{\sigma} l^{2} \sup _{0 \leq s \leq b} E|x(s)-v(s)|_{\mathbb{\sharp}}^{2},
\end{aligned}
$$

where we have used the fact that $u_{0}=v_{0}=0$. Hence,

$$
\left\|\Pi_{1} x-\Pi_{1} v\right\|_{b}^{2} \leq L\|u-v\|_{b}^{2}
$$

so, $\Pi_{1}$ is a contraction by $(23)$.

Step 3. We show that the operator $\Pi_{2}$ is compact. Let $q>0$ be such that $\Pi_{2}\left(B_{q}\right) \subset B_{q}$. The proof will be divided into the following claims. 
Claim $1 . \Pi_{2}$ maps bounded sets into bounded sets in $B_{q}$.

Indeed, it is enough to show that there exists a positive constant $\Delta$ such that for each $t \in J, z \in B_{q}$, we have $\left\|\Pi_{2} z\right\|_{b}^{2} \leq$ $\Delta$. If $z \in B_{q}$, from (32), it follows that

$$
\left\|z_{t}+\widetilde{\phi}_{t}\right\|_{\mathscr{C}_{h}}^{2} \leq 4 l^{2}\left(q+M^{2} E|\phi(0)|_{\mathbb{H}}^{2}\right)+4\|\phi\|_{\mathscr{C}_{h}}^{2}:=q^{\prime}
$$

By the similar argument as before, we get

$$
\begin{aligned}
E\left|\Pi_{2} z(t)\right|_{\mathbb{W}}^{2} \leq & \left(\frac{M \alpha}{\Gamma(1+\alpha)}\right)^{2} \int_{0}^{t}(t-s)^{2(\alpha-1)} d s \\
& \times \int_{0}^{t} E\left|\int_{0}^{s} \sigma\left(s, \tau, \widetilde{\phi}_{\tau}+z_{\tau}\right) d W(\tau)\right|_{\varpi}^{2} d s \\
\leq & \left(\frac{M \alpha}{\Gamma(1+\alpha)}\right)^{2} \frac{\operatorname{Tr} Q b^{2 \alpha-1}}{2 \alpha-1} \Xi\left(q^{\prime}\right) \int_{0}^{t} m(s) d s \\
\leq & \left(\frac{M \alpha}{\Gamma(1+\alpha)}\right)^{2} \frac{\operatorname{Tr} Q b^{2 \alpha-1}}{2 \alpha-1} \Xi\left(q^{\prime}\right) \int_{0}^{b} m(s) d s \\
& :=\Delta .
\end{aligned}
$$

Therefore, for each $z \in B_{q}$, it holds that $\left\|\Pi_{2} z\right\|_{b}^{2} \leq \Delta$.

Claim 2. The set $\left\{\Pi_{2} z, z \in B_{q}\right\}$ is an equicontinuous family of functions on $J$. Let $0<\epsilon<t<b$ and $\delta>0$ such that $\left\|T_{\alpha}\left(s_{1}\right)-T_{\alpha}\left(s_{2}\right)\right\|<\epsilon$, for every $s_{1}, s_{2} \in J$ with $\left|s_{1}-s_{2}\right|<\delta$. For $z \in B_{q}, 0<|h|<\delta, t+h \in J$, we have

$$
\begin{aligned}
& E\left|\Pi_{2} z(t+h)-\Pi_{2} z(t)\right|_{\mathbb{H}}^{2} \\
& =E \mid \int_{0}^{t+h}(t+h-s)^{\alpha-1} T_{\alpha}(t+h-s) \\
& \times\left[\int_{0}^{s} \sigma\left(s, \tau, \tilde{\phi}_{\tau}+z_{\tau}\right) d W(\tau)\right] d s \\
& -\int_{0}^{t}(t-s)^{\alpha-1} T_{\alpha}(t-s) \\
& \times\left.\left[\int_{0}^{s} \sigma\left(s, \tau, \tilde{\phi}_{\tau}+z_{\tau}\right) d W(\tau)\right] d s\right|_{\mathbb{H}} ^{2} \\
& \leq 3 E \mid \int_{t}^{t+h}(t+h-s)^{\alpha-1} T_{\alpha}(t+h-s) \\
& \times\left.\left[\int_{0}^{s} \sigma\left(s, \tau, \tilde{\phi}_{\tau}+z_{\tau}\right) d W(\tau)\right] d s\right|_{\mathbb{H}} ^{2} \\
& +3 E \mid \int_{0}^{t}\left[(t+h-s)^{\alpha-1}-(t-s)^{\alpha-1}\right] T_{\alpha}(t+h-s) \\
& \times\left.\left[\int_{0}^{s} \sigma\left(s, \tau, \widetilde{\phi}_{\tau}+z_{\tau}\right) d W(\tau)\right] d s\right|_{\mathbb{H}} ^{2}
\end{aligned}
$$

$$
\begin{array}{r}
+3 E \mid \int_{0}^{t}(t-s)^{\alpha-1}\left[T_{\alpha}(t+h-s)-T_{\alpha}(t-s)\right] \\
\times\left.\left[\int_{0}^{s} \sigma\left(s, \tau, \tilde{\phi}_{\tau}+z_{\tau}\right) d W(\tau)\right] d s\right|_{\oplus} ^{2} .
\end{array}
$$

Applying assumption $\left(A_{2}\right)$, Hölder inequality, together with Lemma 7, we get

$$
\begin{aligned}
& E\left|\Pi_{2} z(t+h)-\Pi_{2} z(t)\right|_{\mathbb{H}}^{2} \\
& \leq 3 Z(\alpha) \int_{t}^{t+h}(t+h-s)^{2(\alpha-1)} d s \\
& \times \int_{t}^{t+h} E\left|\int_{0}^{s} \sigma\left(s, \tau, \widetilde{\phi}_{\tau}+z_{\tau}\right) d W(\tau)\right|_{\square}^{2} d s \\
& +3 Z(\alpha) \int_{0}^{t}\left[(t+h-s)^{\alpha-1}-(t-s)^{\alpha-1}\right]^{2} d s \\
& \times \int_{0}^{t} E\left|\int_{0}^{s} \sigma\left(s, \tau, \widetilde{\phi}_{\tau}+z_{\tau}\right) d W(\tau)\right|_{\oplus}^{2} d s \\
& +3 \epsilon \int_{0}^{t}(t-s)^{2(\alpha-1)} d s
\end{aligned}
$$

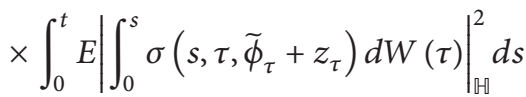

$$
\begin{aligned}
& \leq 3 Z(\alpha) \frac{h^{2 \alpha-1}}{2 \alpha-1} \int_{t}^{t+h} \operatorname{Tr} Q M_{\sigma} m(s) \Xi\left(q^{\prime}\right) d s \\
& +3 Z(\alpha) \int_{0}^{t}\left[(t+h-s)^{\alpha-1}-(t-s)^{\alpha-1}\right]^{2} d s \\
& \times \int_{0}^{t} \operatorname{Tr} Q M_{\sigma} m(s) \Xi\left(q^{\prime}\right) d s \\
& +3 \epsilon \int_{0}^{t}(t-s)^{2(\alpha-1)} d s \int_{0}^{t} \operatorname{Tr} Q M_{\sigma} m(s) \Xi\left(q^{\prime}\right) d s,
\end{aligned}
$$

with $Z(\alpha)=(M \alpha / \Gamma(1+\alpha))^{2}$. Therefore, for $\epsilon$ sufficiently small, the right hand side of (51) tends to zero as $h \rightarrow 0$. Thus, the set $\left\{\Pi_{2} z, z \in B_{q}\right\}$ is equicontinuous by assumption $\left(A_{1}\right)$.

Claim 3. $\Pi_{2}$ maps $B_{q}$ into a precompact set in $B_{q}$. Let $0<t \leq$ $b$ be fixed and $\epsilon$ a real number satisfying $0<\epsilon<t$. For $\delta>0$, define an operator $\Pi_{2}^{\epsilon, \delta}$ on $B_{q}$ by

$$
\begin{aligned}
\left(\Pi_{2}^{\epsilon, \delta} z\right)(t) & \\
=\alpha \int_{0}^{t-\epsilon} \int_{\delta}^{\infty} & \theta(t-s)^{\alpha-1} \eta_{\alpha}(\theta) S\left((t-s)^{\alpha} \theta\right) \\
& \times\left[\int_{0}^{s} \sigma\left(s, \tau, \widetilde{\phi}_{\tau}+z_{\tau}\right) d W(\tau)\right] d \theta d s
\end{aligned}
$$




$$
\begin{aligned}
=S\left(\epsilon^{\alpha} \delta\right) \alpha \int_{0}^{t-\epsilon} \int_{\delta}^{\infty} & \theta(t-s)^{\alpha-1} \eta_{\alpha}(\theta) S \\
\times & \left((t-s)^{\alpha} \theta-\epsilon^{\alpha} \delta\right) \\
\times & {\left[\int_{0}^{s} \sigma\left(s, \tau, \tilde{\phi}_{\tau}+z_{\tau}\right) d W(\tau)\right] d \theta d s . }
\end{aligned}
$$

Since $S(t) \sigma\left(s, \tau, \widetilde{\phi}_{\tau}+z_{\tau}\right)$ belongs to the compact set $\mho$, the set $\left.\left\{\Pi_{2}^{\epsilon, \delta} z\right)(t), z \in B_{q}\right\}$ is precompact in $\mathbb{H}$ for every $\epsilon \in(0, t)$, $\delta>0$. Moreover, for each $z \in B_{q}$, we have

$$
\begin{gathered}
E\left|\left(\Pi_{2} z\right)(t)-\left(\Pi_{2}^{\epsilon, \delta} z\right)(t)\right|_{\mathbb{t}}^{2} \\
\leq 2 \alpha^{2} E \mid \int_{0}^{t} \int_{0}^{\delta} \theta(t-s)^{\alpha-1} \eta_{\alpha}(\theta) S\left((t-s)^{\alpha} \theta\right) \\
\quad \times\left.\left[\int_{0}^{s} \sigma\left(s, \tau, \tilde{\phi}_{\tau}+z_{\tau}\right) d W(\tau)\right] d \theta d s\right|_{\mathbb{⿴}} ^{2} \\
+2 \alpha^{2} E \mid \int_{t-\epsilon}^{t} \int_{\delta}^{\infty} \theta(t-s)^{\alpha-1} \eta_{\alpha}(\theta) S\left((t-s)^{\alpha} \theta\right) \\
\times\left.\left[\int_{0}^{s} \sigma\left(s, \tau, \tilde{\phi}_{\tau}+z_{\tau}\right) d W(\tau)\right] d \theta d s\right|_{\mathbb{t}} ^{2}
\end{gathered}
$$$$
=J_{1}+J_{2} \text {. }
$$

An application of Hölder inequality together with Burkhölder-Davis-Gundy's inequality, it follows that

$$
\begin{aligned}
J_{1} \leq & 2 \alpha^{2} M^{2} \int_{0}^{t}(t-s)^{2(\alpha-1)} E \\
& \times\left|\int_{0}^{s} \sigma\left(s, \tau, \tilde{\phi}_{\tau}+z_{\tau}\right) d W(\tau)\right|_{\mathbb{\sharp}}^{2} d s \\
& \times \int_{0}^{t}\left|\int_{0}^{\delta} \theta \eta_{\alpha}(\theta) d \theta\right|^{2} d s \\
\leq & 2 \alpha^{2} M^{2} b \int_{0}^{t}(t-s)^{2(\alpha-1)} \operatorname{Tr} Q \\
& \times\left.\left.\int_{0}^{s} E\right|_{\sigma}\left(s, \tau, \tilde{\phi}_{\tau}+z_{\tau}\right)\right|_{\mathscr{L}_{2}^{0}} ^{2} d \tau d s\left|\int_{0}^{\delta} \theta \eta_{\alpha}(\theta) d \theta\right|^{2} \\
\leq & 2 \alpha^{2} M^{2} b \int_{0}^{t} \operatorname{Tr} Q \Xi\left(q^{\prime}\right) m(s)(t-s)^{2(\alpha-1)} d s \\
& \times\left|\int_{0}^{\delta} \theta \eta_{\alpha}(\theta) d \theta\right|^{2}, \\
J_{2} \leq & 2 \alpha^{2} M^{2} \int_{t-\epsilon}^{t}(t-s)^{2(\alpha-1)} E \\
&
\end{aligned}
$$

$$
\begin{aligned}
& \times \int_{t-\epsilon}^{t}\left|\int_{0}^{\infty} \theta \eta_{\alpha}(\theta) d \theta\right|^{2} d s \\
\leq & \frac{2 \alpha^{2} M^{2} \epsilon}{\Gamma^{2}(1+\alpha)} \int_{t-\epsilon}^{t} \operatorname{Tr} Q \Xi\left(q^{\prime}\right) m(s)(t-s)^{2(\alpha-1)} d s .
\end{aligned}
$$

From (54), we deduce that for each $z \in B_{q}$,

$$
E\left|\left(\Pi_{2} z\right)(t)-\left(\Pi_{2}^{\epsilon, \delta} z\right)(t)\right|_{\mathbb{\sharp}}^{2} \longrightarrow 0 \quad \text { as } \epsilon \longrightarrow 0^{+}, \delta \longrightarrow 0^{+} .
$$

Therefore, there are relatively compact sets arbitrary close to the set $\left\{\Pi_{2}(t), z \in B_{q}\right\}$; hence, the set $\left\{\left(\Pi_{2} z\right)(t), z \in B_{q}\right\}$ is also precompact in $B_{q}$.

Thus, from Arzelá-Ascoli theorem together with assumptions on $f$ and $\sigma$, we conclude that $\Pi_{2}$ is a compact operator. Therefore, $\Pi$ is a condensing map on $B_{q}$. This completes the proof.

Remark 11. In order to describe various real-world problems in physical and engineering sciences subject to abrupt changes at certain instants during the evolution process, impulsive differential equations have been used to model the system. The technique used here can be extended to establish the controllability of neutral fractional stochastic integro-differential systems with impulsive effect and infinite delay. The controllability result can be obtained by suitably introducing the impulsive effects defined in [19].

\section{Conclusions}

In this paper, we have studied the controllability of fractional neutral stochastic integro-differential systems with infinite delay in an abstract space. Through fractional calculus and Sadovskii's fixed point principle, we have investigated the sufficient conditions for the controllability of the system considered.

\section{Acknowledgments}

This work is supported by the National Natural Science Foundation of China (no. 11171062), Innovation Program of Shanghai Municipal Education Commission (no. 12ZZ063), Natural Science Foundation of Anhui Province (no. 1308085QA14), and Key Natural Science Foundation of Anhui Education Commission (no. KJ2013A133).

\section{References}

[1] M. Benchohra, J. Henderson, S. K. Ntouyas, and A. Ouahab, "Existence results for fractional order functional differential equations with infinite delay," Journal of Mathematical Analysis and Applications, vol. 338, no. 2, pp. 1340-1350, 2008.

[2] C. S. Goodrich, "Existence of a positive solution to systems of differential equations of fractional order," Computers \& Mathematics with Applications, vol. 62, no. 3, pp. 1251-1268, 2011.

[3] A. A. Kilbas, H. M. Srivastava, and J. J. Trujillo, Theory and Applications of Fractional Differential Equations, vol. 204 of North-Holland Mathematics Studies, Elsevier Science B.V., Amsterdam, The Netherlands, 2006. 
[4] I. Podlubny, Fractional Differential Equations, vol. 198 of Mathematics in Science and Engineering, Academic Press, San Diego, Calif, USA, 1999.

[5] J. Wang and Y. Zhou, "A class of fractional evolution equations and optimal controls," Nonlinear Analysis: Real World Applications, vol. 12, no. 1, pp. 262-272, 2011.

[6] J. Wang, Y. Zhou, and M. Medved, "On the solvability and optimal controls of fractional integrodifferential evolution systems with infinite delay," Journal of Optimization Theory and Applications, vol. 152, no. 1, pp. 31-50, 2012.

[7] Y. Zhou and F. Jiao, "Existence of mild solutions for fractional neutral evolution equations," Computers \& Mathematics with Applications, vol. 59, no. 3, pp. 1063-1077, 2010.

[8] L. Chen, Y. Chai, R. Wu, J. Sun, and T. Ma, "Cluster synchronization in fractional-order complex dynamical networks," Physics Letters A, vol. 376, no. 35, pp. 2381-2388, 2010.

[9] S. S. Delshad, M. M. Asheghan, and M. H. Beheshti, "Synchronization of $\mathrm{N}$-coupled incommensurate fractional-order chaotic systems with ring connection," Communications in Nonlinear Science and Numerical Simulation, vol. 16, no. 9, pp. 3815-3824, 2011.

[10] J. Wang and Y. Zhang, "Network synchronization in a population of star-coupled fractional nonlinear oscillators," Physics Letters A, vol. 374, no. 13-14, pp. 1464-1468, 2010.

[11] R. Zhang, D. Qi, and Y. Wang, "Dynamics analysis of fractional order three-dimensional Hopfield neural network," in Proceedings of the 6th International Conference on Natural Computation (ICNC'10), vol. 6, pp. 3037-3039, Yantai, China, August 2010.

[12] E. Kaslik and S. Sivasundaram, "Nonlinear dynamics and chaos in fractional order neural networks," Neural Networks, vol. 32, pp. 245-256, 2012.

[13] K. Sobczyk, Stochastic Differential Equations with Applications to Physics and Engineering, vol. 40 of Mathematics and its Applications (East European Series), Kluwer Academic, London, UK, 1991.

[14] M. E. Gurtin and A. C. Pipkin, "A general theory of heat conduction with finite wave speeds," Archive for Rational Mechanics and Analysis, vol. 31, no. 2, pp. 113-126, 1968.

[15] J. W. Nunziato, "On heat conduction in materials with memory," Quarterly of Applied Mathematics, vol. 29, pp. 187-204, 1971.

[16] M. M. El-Borai, K. E.-S. El-Nadi, O. L. Mostafa, and H. M. Ahmed, "Volterra equations with fractional stochastic integrals," Mathematical Problems in Engineering, vol. 2004, no. 5, pp. 453-468, 2004.

[17] M. M. El-Borai, "On some stochastic fractional integrodifferential equations," Advances in Dynamical Systems and Applications, vol. 1, no. 1, pp. 49-57, 2006.

[18] J. P. Dauer and N. I. Mahmudov, "Controllability of stochastic semilinear functional differential equations in Hilbert spaces," Journal of Mathematical Analysis and Applications, vol. 290, no. 2, pp. 373-394, 2004.

[19] R. Sakthivel, Y. Ren, and N. I. Mahmudov, "Approximate controllability of second-order stochastic differential equations with impulsive effects," Modern Physics Letters B, vol. 24, no. 14, pp. 1559-1572, 2010.

[20] R. Subalakshmi and K. Balachandran, "Approximate controllability of neutral stochastic integrodifferential systems in Hilbert spaces," Electronic Journal of Differential Equations, vol. 2008, no. 162, pp. 1-15, 2008.

[21] J. Wang and Y. Zhou, "Complete controllability of fractional evolution systems," Communications in Nonlinear Science and Numerical Simulation, vol. 17, no. 11, pp. 4346-4355, 2012.
[22] L. Zhang, Y. Ding, T. Wang, L. Hu, and K. Hao, "Controllability of second-order semilinear impulsivestochastic neutral functional evolution equations," Mathematical Problems in Engineering, vol. 2012, Article ID 748091, 13 pages, 2012.

[23] P. Balasubramaniam and S. K. Ntouyas, "Controllability for neutral stochastic functional differential inclusions with infinite delay in abstract space," Journal of Mathematical Analysis and Applications, vol. 324, no. 1, pp. 161-176, 2006.

[24] P. Balasubramaniam, J. Y. Park, and P. Muthukumar, "Approximate controllability of neutral stochastic functional differential systems with infinite delay," Stochastic Analysis and Applications, vol. 28, no. 2, pp. 389-400, 2010.

[25] Y. Ren, L. Hu, and R. Sakthivel, "Controllability of impulsive neutral stochastic functional differential inclusions with infinite delay," Journal of Computational and Applied Mathematics, vol. 235, no. 8, pp. 2603-2614, 2011.

[26] A. Pazy, Semigroups of Linear Operators and Applications to Partial Differential Equations, vol. 44 of Applied Mathematical Sciences, Springer, New York, NY, USA, 1983.

[27] G. Da Prato and J. Zabczyk, Stochastic Equations in Infinite Dimensions, vol. 44 of Encyclopedia of Mathematics and its Applications, Cambridge University Press, Cambridge, UK, 1992.

[28] S. G. Samko, A. A. Kilbas, and O. I. Marichev, Fractional Integrals and Derivatives: Theory and Applications, Gordon and Breach Science, Yverdon, Switzerland, 1993.

[29] Y. Liu, W. Zhang, and X. Liu, "A sufficient condition for the existence of a positive solution for a nonlinear fractional differential equation with the Riemann-Liouville derivative," Applied Mathematics Letters, vol. 25, no. 11, pp. 1986-1992, 2012.

[30] B. N. Sadovskii, “On a fixed point principle," Functional Analysis and Its Applications, vol. 1, pp. 71-74, 1967.

[31] N. Carimichel and M. D. Quinn, Fixed Point Methods in Nonlinear Control, vol. 75 of Lecture Notes in Controland Information Society, Springer, Berlin, Germany, 1984.

[32] C.-M. Marle, Mesures et Probabilités, Hermann, Paris, France, 1974. 


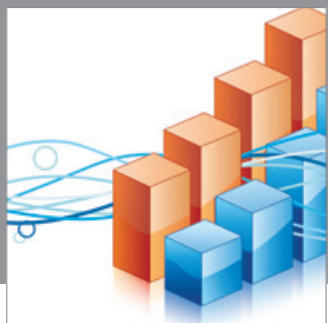

Advances in

Operations Research

mansans

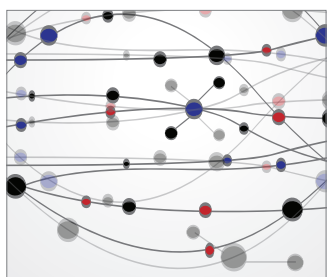

The Scientific World Journal
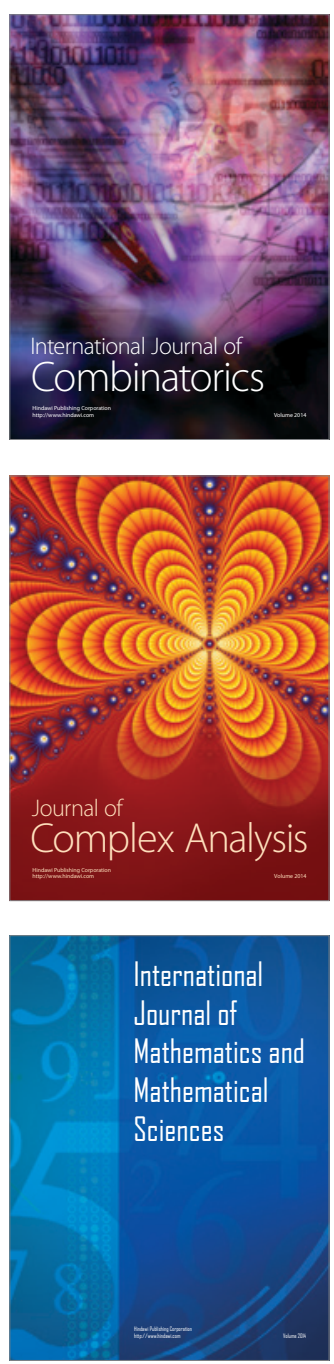
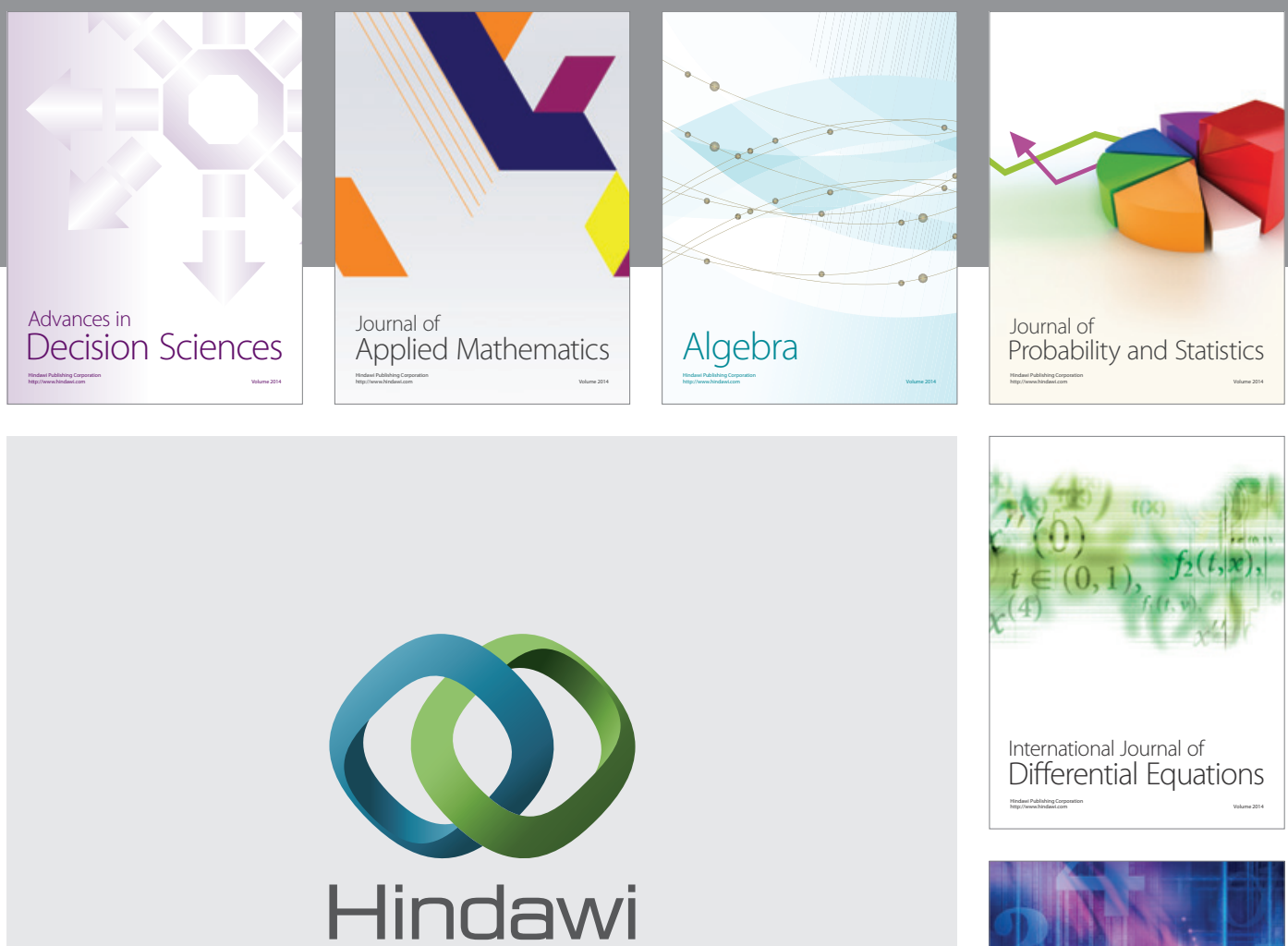

Submit your manuscripts at http://www.hindawi.com
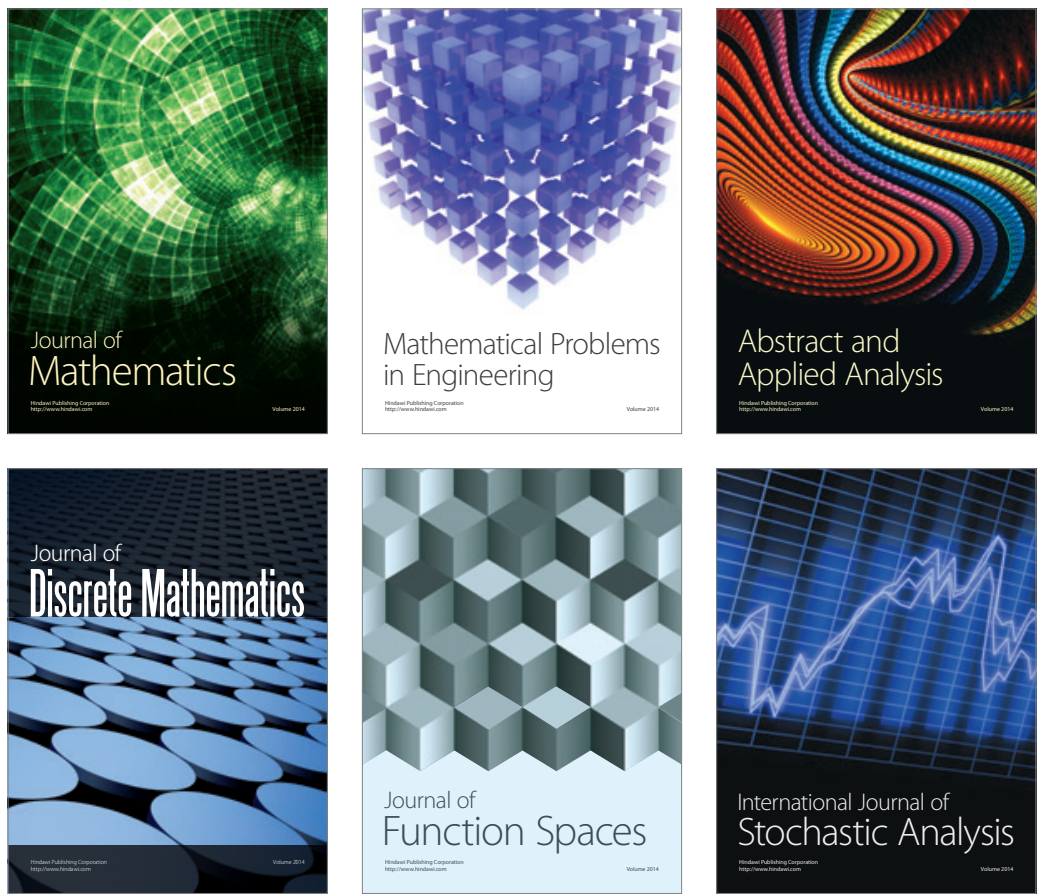

Journal of

Function Spaces

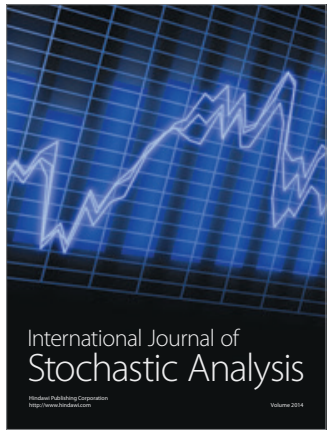

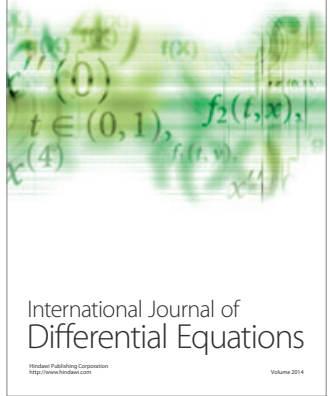
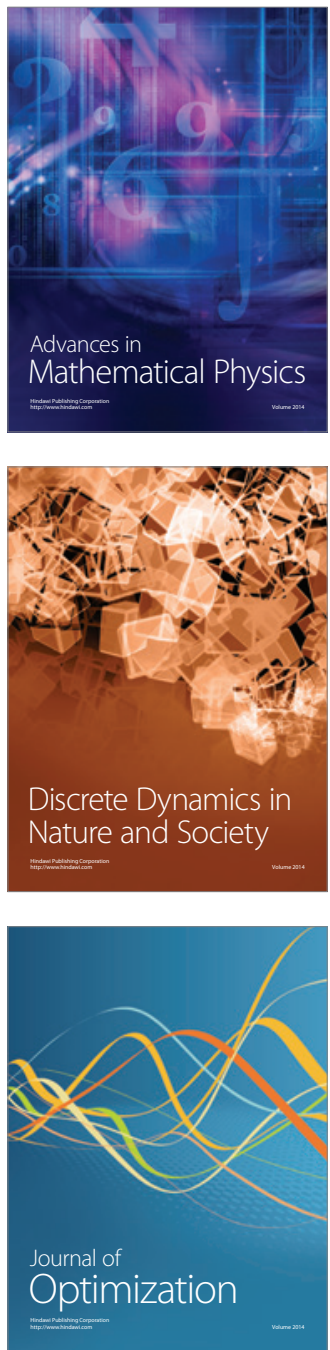